\title{
Monitoring childbirth care in primary health facilities: a validity study in Gombe State, northeastern Nigeria
}

\author{
Antoinette Alas \\ Bhattacharya ${ }^{1}$, Elizabeth \\ Allen², Nasir Umar ${ }^{1}$, Adamu \\ Umar Usman³, Habila Felix ${ }^{4}$, \\ Ahmed Audu', Joanna \\ RM Schellenberg ${ }^{1}$, Tanya \\ Marchant $^{1}$ \\ ${ }^{1}$ Department of Disease Control, \\ London School of Hygiene \& Tropical \\ Medicine, London, UK \\ 2 Department of Medical Statistics, \\ London School of Hygiene \& Tropical \\ Medicine, London, UK \\ ${ }^{3}$ Data Research and Mapping Consult, \\ Abuja, Nigeria \\ ${ }^{4}$ State Primary Health Care Development \\ Agency, Gombe, Nigeria
}

\begin{abstract}
Background Improving the quality of facility-based births is a critical strategy for reducing the high burden of maternal and neonatal mortality and morbidity across all settings. Accurate data on childbirth care is essential for monitoring progress. In northeastern Nigeria, we assessed the validity of childbirth care indicators in a rural primary health care context, as documented by health workers and reported by women at different recall periods.
\end{abstract}

Methods We compared birth observations (gold standard) to: (i) facility exit interviews with observed women; (ii) household follow-up interviews 9-22 months after childbirth; and (iii) health worker documentation in the maternity register. We calculated sensitivity, specificity, and area under the receiver operating curve (AUC) to determine individual-level reporting accuracy. We calculated the inflation factor (IF) to determine population-level validity.

Results Twenty-five childbirth care indicators were assessed to validate health worker documentation and women's self-reports. During exit interviews, women's recall had high validity (AUC $\geq 0.70$ and $0.75<\mathrm{IF}<1.25$ ) for 9 of 20 indicators assessed; six additional indicators met either AUC or IF criteria for validity. During follow-up interviews, women's recall had high validity for one of 15 indicators assessed, placing the newborn skinto-skin; two additional indicators met IF criteria only. Health worker documentation had high validity for four of 10 indicators assessed; three additional indicators met AUC or IF criteria.

Conclusions In addition to standard household surveys, monitoring of facility-based childbirth care should consider drawing from and linking multiple data sources, including routine health facility data and exit interviews with recently delivered women.

\section{Correspondence to:}

Antoinette Bhattacharya Department of Disease Control London School of Hygiene \& Tropical Medicine

Keppel Street

London

W1CE 7HT

United Kingdom

antoinette.bhattacharya@Ishtm.ac.uk
The childbirth process presents a time of great risk of death for women and their newborns [1,2]. Of the estimated 303000 maternal deaths and 2.5 million neonatal deaths that occurred in 2015, 113000 maternal deaths and over 1 million neonatal deaths were attributed to complications from childbirth and the immediate postpartum period $[3,4]$. The distribution of this risk of death is uneven. While $36 \%$ of the world's population lives in sub-Saharan Africa and Southern Asia, these regions account for $86 \%$ of maternal deaths and at least $78 \%$ of the newborn deaths [1-5]. For facility-based births, improving the quality of care for women and newborns especially during the intrapartum period 
is considered one of the most effective strategies for reducing maternal and neonatal mortality and morbidity across all settings [1,6-10].

Global and national monitoring of facility-based care often includes self-reported retrospective data collected in household surveys such as the Demographic and Health Survey (DHS) and Multiple Indicator Cluster Survey (MICS) [11-13]. For population-based coverage estimates of childbirth care, these periodic and nationally representative surveys collect a limited set of data which include maternal background characteristics and birth history, delivery by a skilled birth attendant, and newborn care practices $[14,15]$. A small number of criterion validity studies of childbirth care which measured the extent to which the women's self-reported data at different recall periods align with a gold standard, have demonstrated mixed results on the accuracy of data in household surveys [16-22]. Understanding how best to accurately monitor childbirth care is an emerging research priority and evidence from different contexts is required $[23,24]$.

Routine data can be used to monitor the content of facility-based care, but concerns about completeness, consistency, and accuracy have hampered their use [13]. Most studies on the accuracy of routine data have focused on verifying the aggregate data reported by facilities to higher management levels and comparing these to data documented by health workers [25-30]. However, similar to the population-based surveys, the extent to which the data documented by health workers reflect the "truth" of care is also not well-established [31].

In the high mortality setting of northeastern Nigeria, we assessed the extent to which different data recording methods could contribute to the global- and national-level monitoring of maternal and newborn health. Using direct birth observations as a gold standard, we compared these observations to: (i) facility exit interviews with women after childbirth; (ii) household follow-up interviews with women nine to 22 months after childbirth; and (iii) health worker documentation of childbirth events in the facility maternity register.

\section{METHODS}

\section{Ethical review}

Study approvals were obtained from the London School of Hygiene \& Tropical Medicine (reference 14091) and the Health Research Ethics Committees for Nigeria (reference NHREC/01/01/2007) and Gombe State (reference ADM/S/658/Vol. II/66).

\section{Study setting}

Gombe State, northeastern Nigeria, has high maternal and newborn mortality at 814 per 100000 live births and 35 per 1000 live births, respectively; nationally, maternal mortality estimates are also 814 per 100000 live births and neonatal mortality estimates are 39 per 1000 live births $[3,4,14,15]$. Gombe is predominantly rural and $44 \%$ of the population have some primary school education. Most women access maternity care through public facilities. Seventy-two percent of women reported at least one antenatal care visit during their last pregnancy and 29\% gave birth in a health facility [15]. In 2018, over 70\% of facility deliveries took place in rural primary health facilities [32].

\section{Indicator selection}

Twenty-five indicators were selected, focusing on the content of childbirth care (Table 1): skilled birth attendance and companionship during labor and delivery; care for the woman (maternal background characteristics, provider practices and respectful care, clinical care); and care for the newborn (immediate postnatal care and newborn outcomes). To select these indicators, we referred to the Ending Preventable Maternal Mortality and Every Newborn Action Plan strategy documents for priority indicators to monitor progress towards Sustainable Development Goals targets $[33,34]$. We also sought to complement indicators collected in the Nigeria Demographic and Health Survey as well as earlier studies validating childbirth care indicators $[14,16-20]$.

In Gombe, maternity registers defined essential newborn care as the immediate initiation of breastfeeding and the baby being kept warm within 30 minutes of birth [35]. To determine if the maternity register provided a sufficient approximation to globally-defined indicators, we compared the maternity register's essential newborn care data to being kept warm and the initiation of breastfeeding within the first hour 
Table 1. Childbirth care indicators and data recording methods compared with birth observations (gold standard) for validation analyses

\begin{tabular}{|c|c|c|c|c|}
\hline \multirow[b]{2}{*}{ Indicator } & \multirow[b]{2}{*}{ Births observation } & \multicolumn{3}{|c|}{ COMPARISON DATA RECORDING METHOD* } \\
\hline & & $\begin{array}{l}\text { Facility exit } \\
\text { interview }\end{array}$ & $\begin{array}{l}\text { Household } \\
\text { follow-up } \\
\text { interview }\end{array}$ & $\begin{array}{c}\text { Facility } \\
\text { maternity } \\
\text { register }\end{array}$ \\
\hline
\end{tabular}

Skilled birth attendance and companionship during labor and delivery:

\begin{tabular}{|c|c|c|c|c|}
\hline Main provider - doctor, nurse, or midwife & $\mathrm{X}$ & $\mathrm{X}$ & $\mathrm{X}$ & $\mathrm{X}$ \\
\hline More than one provider present at birth & $\mathrm{X}$ & $\mathrm{X}$ & $\mathrm{X}$ & \\
\hline Support person present at birth & $\mathrm{X}$ & $\mathrm{X}$ & $\mathrm{X}$ & \\
\hline \multicolumn{5}{|l|}{ Care for the woman: } \\
\hline \multicolumn{5}{|l|}{ Maternal background $\uparrow$ : } \\
\hline Age at delivery (adolescent births) & $\mathrm{X}$ & & & $\mathrm{x}$ \\
\hline Prior parity (prior parity, 4 or more births) & $\mathrm{X}$ & & & $\mathrm{X}$ \\
\hline \multicolumn{5}{|l|}{ Provider practices and respectful care: } \\
\hline Woman allowed to move and change position during labor & $\mathrm{X}$ & $\mathrm{X}$ & & \\
\hline Woman allowed to drink liquids and eat during labor & $\mathrm{X}$ & $\mathrm{X}$ & & \\
\hline Woman allowed to deliver in preferred position & $\mathrm{X}$ & $\mathrm{X}$ & & \\
\hline Woman allowed to have a support person at birth & $\mathrm{X}$ & $\mathrm{X}$ & $\mathrm{X}$ & \\
\hline Birth attendant washed hands with soap before examinations & $\mathrm{X}$ & $\mathrm{X}$ & $\mathrm{X}$ & \\
\hline Birth attendant wore gloves during examinations & $\mathrm{X}$ & $\mathrm{X}$ & $\mathrm{X}$ & \\
\hline Partograph used to monitor labor and delivery & $\mathrm{X}$ & & & $\mathrm{X}$ \\
\hline \multicolumn{5}{|l|}{ Clinical care: } \\
\hline Blood pressure taken - initial client assessment & $\mathrm{X}$ & $\mathrm{X}$ & $\mathrm{X}$ & \\
\hline Episiotomy performed & $\mathrm{X}$ & $\mathrm{X}$ & $\mathrm{X}$ & \\
\hline $\begin{array}{l}\text { Prophylactic uterotonic administered during third stage of labor to } \\
\text { prevent postpartum hemorrhage }\end{array}$ & $\mathrm{X}$ & $\mathrm{X}$ & $\mathrm{X}$ & $\mathrm{x}$ \\
\hline
\end{tabular}

\section{Care for the newborn:}

Immediate postnatal care:

Mother and newborn kept in same room after delivery

Newborn immediately dried with a towel

Newborn immediately placed skin-to-skin

Immediate initiation of breastfeeding

Essential newborn care $\ddagger$

Chlorhexidine applied to newborn's cord to prevent infection

Baby weighed at birth

Newborn outcomes:

Low birthweight, $<2500 \mathrm{~g}^{\dagger} \quad \mathrm{X}$

Pre-term birth $\quad \mathrm{X}$

Stillbirth, fresh or macerated

Total indicators

$\begin{array}{ll}X & X \\ X & X \\ X & X \\ X & X \\ X & X \\ X & \\ X & \end{array}$

$\mathrm{X} \quad \mathrm{X}$

$\mathrm{X} \quad \mathrm{X}$

$\mathrm{x} \quad \mathrm{x}$

$\mathrm{X} \quad \mathrm{X}$

$\mathrm{X} \quad \mathrm{X}$

$\mathrm{X}$

$\mathrm{X} \quad \mathrm{X} \quad \mathrm{X}$

\begin{tabular}{lll}
$X$ & $X$ & $X$ \\
$X$ & & $X$ \\
$X$ & 20 & $X$ \\
\hline 25 & 15 & 10
\end{tabular}

*Observed women were interviewed before discharge from the facility (exit interview) and at home nine to 22 mo after childbirth (follow-up interview) Health workers documented childbirth events in facility maternity registers.

$†$ For validation analyses, the following indicators were converted into binary variables: age at delivery (adolescent births); prior parity (prior parity, four or more births); and baby's birthweight (low birthweight, $<2500 \mathrm{~g}$ ).

¥In the facility maternity register, essential newborn care is a composite indicator for (i) immediate initiation of breastfeeding and (ii) baby kept warm.

of birth [34]. For validation analyses, the following indicators were converted into binary variables: maternal age at delivery (adolescent births); prior parity (prior parity, four or more births); and baby's birthweight (low birthweight, <2500 g).

\section{Study sites and data sources}

As part of an initiative to improve care in Gombe State, data were collected between 2016-2018, including facility-based birth observations [36]. A summary of each data recording method is provided in Figure 1 ; detailed descriptions follow.

\section{Birth observations}

Starting in June 2016, five rounds of birth observations took place in 10 primary health facilities. Each round took place roughly every six months and lasted three weeks. To select the facilities for birth observations, a state-wide random sample of 107 facilities was drawn in November 2015 from approximate- 


\begin{tabular}{|c|c|c|c|c|c|c|}
\hline & $\begin{array}{l}\text { June } \\
2016\end{array}$ & $\begin{array}{l}\text { March } \\
2017\end{array}$ & $\begin{array}{l}\text { August } \\
2017\end{array}$ & $\begin{array}{l}\text { March } \\
2018\end{array}$ & $\begin{array}{l}\text { August } \\
2018\end{array}$ & Total \\
\hline Birth observations (gold standard) & 373 & 385 & 361 & 416 & 354 & 1889 \\
\hline Exit interview after observed childbirth* & 340 & 360 & 344 & 394 & 336 & 1774 \\
\hline Follow-up interview 9-22 months after observed childbirtht & $4-$ & -1 & --- & 445 & & 445 \\
\hline Facility maternity register & & 365 & 358 & 403 & 347 & 1474 \\
\hline
\end{tabular}

Figure 1. Data recording methods, data collection rounds, and the number of women observed and interviewed. * Of the 1889 women observed, 115 (6\%) did not participate in an exit interview: 11 (0.5\%) were discharged with their newborn and refused to be interviewed; 104 (5.5\%) women were not interviewed (21 were referred to another facility; 61 still births with 1 maternal death; 22 newborn deaths). †A total of 445 women were followed up at home in March 2018, 9-22 months after their observed childbirth: 147 women from deliveries in June $2016 ; 146$ women from deliveries in March 2017; 152 women from deliveries in August 2017.

ly 500 government-owned primary health facilities. The maternity registers were reviewed to determine the volume of births occurring in the previous six months. The 10 facilities with the highest number of births were selected for birth observations [37]. An average of 15.7 births (standard deviation SD =12.0) occurred per month in the 10 primary health facilities, compared to the state-level average of 4.3 births $(\mathrm{SD}=6.3)$ per month in primary health facilities [38].

All women attending the facility for delivery were invited to participate, excluding women admitted for monitoring before the onset of labor. Women were given a description of the study and the procedures, including the right to withdraw participation at any time. A trained observer (local midwives, not employees of the assigned facility) stayed in the same room to continuously document labor and delivery processes through the first hour after birth, using a structured checklist. Labor and delivery took place in the same room. The mother and newborn were usually kept together until discharged from the facility.

Two observers and one clinical supervisor were assigned per facility to work in shifts and cover all deliveries. Although observers were trained midwives, they had no legal right to intervene in clinical care during the observation period because they were not employed in the same facilities where they were doing the observations. At all times during the observation, the observer prioritized safety of the mother and newborn over data collection; protocols were established on how to seek help in the event of any life-threatening event. Priorities for the supervisor were (i) to ensure that consenting procedures were carried out; (ii) to observe data collection and carry out interrater reliability checks; (iii) to assist in the case of a query from facility employees or from clients and families; (iv) to collect and check digital data at the end of each day.

Before each round, observers underwent four days of practical training to conduct unobtrusive observations, train on safety and confidentiality protocols, and ensure consistency of rating between observers. Observations were recorded onto a Lenovo A3300 tablet using CSPro version 7.0 (United States Census Bureau and ICF Macro, Suitland, MD, USA). Each observed woman was assigned a unique observation number to facilitate linking information to other data sets.

\section{Facility maternity registers}

Following the birth observation, regardless of newborn outcome, the observer extracted data about the woman from the maternity register. Data extraction took place on the same day as the observed birth after the first hour of birth. Data were directly entered into the tablet.

\section{Facility exit interviews}

Women were usually discharged within 24 hours of delivery. Each observed woman leaving the facility with a live newborn was invited to participate in an exit interview. The exit interview covered information recorded during the observation and harmonized with questions asked in the DHS and MICS. Each interview was conducted in Hausa by a member of the observation team assigned to the facility. Interview questions are available in Table S1 in Online Supplementary Document. 


\section{Household follow-up interviews, nine to 22 months after childbirth}

In addition to recall during exit interviews, it was of interest to understand the validity of women's recall in the context of household surveys, such as DHS and MICS. For this purpose, we conducted household-level follow-up interviews with a subset of the observed women to recall childbirth events. To represent a range of recall periods that may be encountered during a household survey, in March 2018 we selected approximately 150 women from each of the first three rounds of birth observations which occurred in June 2016 (22 months recall), March 2017 (15 months recall), and August 2017 (9 months recall); this selection was done by a simple random sample of a de-identified list of women observed per round. Each interview was conducted in Hausa and the women were asked the same questions as in the exit interview.

\section{Sample size}

To estimate the sample size, 50\% prevalence from clinical observations (gold standard) was set for all indicators as we expected variability in the frequency of indicators. Sensitivity was set at $60 \% \pm 7 \%$ precision and specificity at $70 \% \pm 7 \%$ precision. Type 1 error was set at 0.05 , assuming a normal approximation to a binomial distribution. Thus, a minimum sample size of 400 was required for observed women at exit interviews, at follow-up interviews, and in the maternity register.

\section{Analysis}

To combine the data from five rounds of data collection, we tested for marginal homogeneity using Yang's chi-square test for clustered binary matched pair data using the clust.bin.pair package in R $[39,40]$. Of the 45 matched pairs analyzed (see Table 1), one indicator showed evidence of clustering across time when comparing birth observations and women's self-reports at exit and follow-up interviews: birth attendant washed hands with soap before examinations. Given the number of matched pairs analyzed, we considered there to be sufficient evidence that the data collection rounds could be combined.

Validation analyses were performed using Stata 14.2 (Stata Corp, College Station, TX, USA) [41]. Using birth observations as the gold standard, we assessed each indicator's validity at the individual- and population-level.

To measure individual-level reporting accuracy, we constructed three two-by-two tables for each indicator which compared the birth observation to each data recording method [16,18-20,23]. Missing and "don't know" responses were excluded from the two-by-two tables. We calculated percent agreement between the birth observation and each data recording method.

For two-by-two tables with at least five observations per cell, we calculated the sensitivity (true positive rate) and specificity (true negative rate) for each indicator. We quantified the area under the receiver operating characteristic curve (AUC) and estimated 95\% confidence intervals (CI) assuming a binomial distribution. AUC values range from 0 to 1 , with 0.5 representing a random guess and 1 representing complete accuracy. An AUC value of 0.7 or higher was chosen as the cutoff criteria for high individual-level reporting accuracy [23].

To measure the population-level validity, we calculated each indicator's inflation factor (IF), which is the ratio of the estimated population-based survey prevalence to the gold standard's prevalence. The IF reflects the degree to which an indicator would be over- or under-estimated in a population-based survey. To estimate the population-based survey prevalence, we used the following equation [42]: estimated population survey prevalence $=($ gold standard prevalence $\times$ sensitivity $)+[(1-$ gold standard prevalence $) \times(1-$ specificity)]. An IF value between 0.75 and 1.25 was the chosen cut-off criteria for low population-level bias [23].

\section{RESULTS}

\section{Sample description}

Characteristics of the women observed during childbirth are presented in Table 2. Women's age ranged from 15 to 47 years, with a median age of 24 years (interquartile range $(I Q R)=20-28$ ). Forty-four percent of women had at least 4 prior deliveries, $47 \%$ of women had no formal education, and $99 \%$ were married.

For each indicator and data recording method: indicator prevalence, "don't know" responses, percent agreement with gold standard, sensitivity, specificity, AUC, and IF values are summarized in Table 3. 
Table 2. Characteristics of women observed during childbirth

\begin{tabular}{|c|c|}
\hline & $\begin{array}{c}\text { NumBER of } \\
\text { WOMEN, } \mathbf{N}(\%), \\
\mathbb{N}=1774^{*}\end{array}$ \\
\hline \multicolumn{2}{|l|}{ Data collection round: } \\
\hline June 2016 & $340(19)$ \\
\hline March 2017 & $360(20)$ \\
\hline August 2017 & $344(19)$ \\
\hline March 2018 & $394(22)$ \\
\hline August 2018 & $336(19)$ \\
\hline \multicolumn{2}{|l|}{ Age of client at delivery: $\dagger$} \\
\hline $15-19$ & $351(20)$ \\
\hline $20-24$ & $600(34)$ \\
\hline $25-29$ & $402(23)$ \\
\hline $30-34$ & $243(14)$ \\
\hline $35-39$ & $126(7)$ \\
\hline $40+$ & $47(3)$ \\
\hline \multicolumn{2}{|l|}{ Prior parity: } \\
\hline 0 & $41(2)$ \\
\hline 1 & $355(20)$ \\
\hline 2 & $339(19)$ \\
\hline 3 & $255(14)$ \\
\hline 4 or more & $779(44)$ \\
\hline \multicolumn{2}{|l|}{ Educational attainment: } \\
\hline None & $827(47)$ \\
\hline Primary & $412(23)$ \\
\hline Secondary & $490(28)$ \\
\hline Higher & $45(3)$ \\
\hline \multicolumn{2}{|l|}{ Marital status: } \\
\hline Single, never married & $12(1)$ \\
\hline Married & $1759(99)$ \\
\hline Widowed & $3(0)$ \\
\hline \multicolumn{2}{|l|}{ Time of delivery:§ } \\
\hline Day, 8:00am-6:59pm & $1038(59)$ \\
\hline Night, 7:00pm-7:59am & $715(40)$ \\
\hline \multicolumn{2}{|l|}{ Day of delivery:\| } \\
\hline Weekday & $1194(67)$ \\
\hline Weekend & $567(32)$ \\
\hline \multicolumn{2}{|l|}{ Main provider during labor and delivery:IL } \\
\hline Doctor, nurse, or midwife & $184(10)$ \\
\hline Community health extension worker, junior CHEW & $690(39)$ \\
\hline Hospital assistant & $387(22)$ \\
\hline Other facility staff & $461(26)$ \\
\hline Other non-staff, including traditional birth attendant & $51(3)$ \\
\hline \multicolumn{2}{|c|}{$\begin{array}{l}\text { CHEW - community health extension worker } \\
\text { "Distribution of characteristics based on the } 1774 \text { respondents during } \\
\text { exit interviews. Percentages do not always add up to } 100 \% \text { due to } \\
\text { rounding, missing responses (up to } 1.1 \%) \text {, and "don't know" respons- } \\
\text { es ( } 0.2 \%) \text {. } \\
\text { †'Age of client at delivery" had } 1(0.1 \%) \text { missing response and } 4 \\
\text { (0.2\%) "don't know" responses. } \\
\text { \$"Prior parity" had } 6(0.3 \%) \text { missing responses. } \\
\text { \&"Time of delivery" had } 19(1.1 \%) \text { missing responses. } \\
\text { "“Day of delivery" had } 13(0.7 \%) \text { missing responses. } \\
\text { q"Main provider during labor and delivery" had } 1(0.1 \%) \text { missing } \\
\text { response. }\end{array}$} \\
\hline
\end{tabular}

maintain the low population-level bias.
Figure 2 presents a summary of the validity criteria met across data recording methods.

"Don't know" responses, which indicate the extent to which recall may or may not be possible, were greater than $5 \%$ for: birth attendant washed hands with soap before examinations (exit and follow-up); baby weighed at birth (exit and follow-up); and low birthweight (exit only). Health workers documented in maternity registers most frequently for: baby weighed at birth (99\% completeness), maternal age at delivery (97\%), and prior parity (97\%). Documentation was least frequent for the composite indicator essential newborn care (82\% completeness) and pre-term birth (77\%).

\section{Skilled birth attendance and companionship during labor and delivery}

Health worker documentation of the main provider's cadre had high overall validity, meaning AUC $\geq 0.70$ for high individual-level accuracy and $0.75<\mathrm{IF}<1.25$ for low population-level bias. During exit interviews, women's recall had high overall validity for the presence of more than one provider at birth and high individual-level accuracy for the main provider's cadre and the presence of a support person during labor and delivery. During follow-up, women's recall for these three indicators met neither validity criteria.

\section{Care for the woman}

Health worker documentation in maternity registers had high overall validity for maternal age at delivery and prior parity and high individual-level accuracy for reporting the use of a partograph. While there was insufficient variation in responses for validation analysis, health worker documentation had near complete agreement with the gold standard for the administration of a prophylactic uterotonic.

During exit interviews, women's recall on four provider respectful care indicators met at least one validity criteria, with high overall validity for two indicators: allowed to move and change positions during labor and allowed to have a support person during labor and delivery. During follow-up, women's recall of being allowed to have a support person maintained low population-level bias only.

During exit interviews, women's report of clinical care received had high overall validity for having her blood pressure taken before delivery and low population-level bias only for the administration of prophylactic uterotonic. During follow-up, only administration of a prophylactic uterotonic was able to

\section{Care for the newborn}

For two indicators requiring the mother's involvement, immediate initiation of breastfeeding and placing the newborn skin-to-skin, women's recall during exit interviews had high overall validity. During follow-up, women's recall of her baby being placed skin-to-skin maintained high overall validity, whereas recall of immediate breastfeeding met neither validity criteria. Health worker documentation of these practices as a composite indicator of essential newborn care met neither validity criteria; health workers documented a 95\% prevalence for being kept warm and initiation of breastfeeding within 30 minutes of birth whereas birth observations documented 39\% prevalence for these practices within one hour of birth. 


\section{Birth attendance and companionship during labor and delivery}

Exit interviews
Follow-up interviews
Maternity register

\section{Main provider - doctor, nurse, or midwife \\ More than one provider present at birth \\ Support person present at birth}

\begin{tabular}{|c|c|}
\hline AUC \\
\hline AUC \\
\hline AUC \\
\hline
\end{tabular}

\section{Exit}

Care for the woman

\section{interviews}

no criteria met

no criteria met

no criteria met

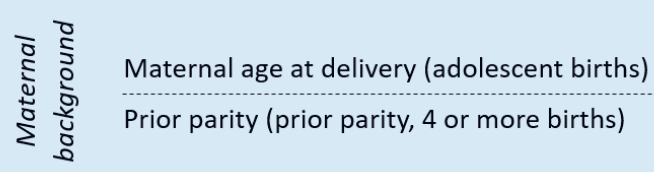

Follow-up interviews

\begin{tabular}{|c|c|c|c|c|c|}
\hline \multirow{6}{*}{ 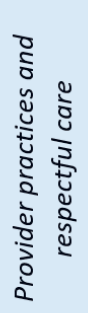 } & Woman allowed to move and change position during labor & AUC & IF & & \\
\hline & \multicolumn{2}{|l|}{ Woman allowed to drink liquids and eat during labor } & \multicolumn{3}{|l|}{ IF } \\
\hline & Woman allowed to deliver in preferred position & AUC & & & \\
\hline & Woman allowed to have a support person at birth & AUC & IF & IF & \\
\hline & Birth attendant washed hands with soap before examinations & \multicolumn{2}{|c|}{$>5 \% \mathrm{dk}$} & \multicolumn{2}{|l|}{$>5 \%$ dk } \\
\hline & Birth attendant wore gloves during examinations & \multicolumn{2}{|c|}{ no criteria met } & $<5 /$ cell & \\
\hline & Partograph used to monitor labor and delivery & & & & AUC \\
\hline \multirow{3}{*}{ 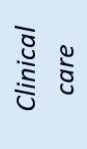 } & Blood pressure taken - initial client assessment & AUC & IF & no criteria met & \\
\hline & Episiotomy performed & \multicolumn{2}{|c|}{$<5$ / cell } & \multicolumn{2}{|l|}{$<5 /$ cell } \\
\hline & Prophylactic uterotonic administered & & IF & IF & $<5 /$ cell \\
\hline
\end{tabular}

\begin{tabular}{|c|c|c|c|c|c|c|c|}
\hline \multicolumn{2}{|c|}{ Care for the newborn } & \multicolumn{2}{|c|}{$\begin{array}{c}\text { Exit } \\
\text { interviews }\end{array}$} & \multicolumn{2}{|c|}{$\begin{array}{l}\text { Follow-up } \\
\text { interviews }\end{array}$} & \multicolumn{2}{|c|}{$\begin{array}{l}\text { Maternity } \\
\text { register }\end{array}$} \\
\hline \multirow{7}{*}{ 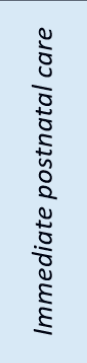 } & Mother and newborn kept in the same room after delivery & & IF & & & & \\
\hline & Essential newborn care* & AUC & IF & no cri & a met & \multicolumn{2}{|c|}{ no criteria met } \\
\hline & Newborn immediately dried with a towel & AUC & IF & \multicolumn{2}{|c|}{$<5 /$ cell } & & \\
\hline & Newborn immediately placed skin-to-skin & AUC & IF & AUC & IF & & \\
\hline & Immediate initiation of breastfeeding & AUC & IF & \multicolumn{2}{|c|}{ no criteria met } & & \\
\hline & Chlorhexidine applied to newborn's cord & AUC & IF & & & & \\
\hline & Baby weighed at birth & \multicolumn{2}{|c|}{$>5 \% \mathrm{dk}$} & \multicolumn{2}{|c|}{$>5 \% \mathrm{dk}$} & & IF \\
\hline \multirow{3}{*}{ 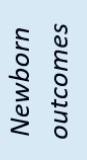 } & Baby's birthweight (low birthweight, <2500 grams) & \multicolumn{2}{|c|}{$>5 \%$ dk } & & & \multicolumn{2}{|c|}{ AUC } \\
\hline & Pre-term birth & & & & & \multicolumn{2}{|c|}{$<5 /$ cell } \\
\hline & Stillbirth, fresh or macerated & & & & & AUC & IF \\
\hline
\end{tabular}

Figure 2. Summary of childbirth care indicator validity criteria across data recording methods. Observed women were interviewed before discharge from the facility (exit interview) and at home nine to 22 months after childbirth (follow-up interview). Health workers documented childbirth events in facility maternity registers. AUC = area under the receiver operating characteristic curve; IF = inflation factor; $>5 \% \mathrm{dk}=>5 \%$ "don't know" responses; $<5 /$ cell = less than 5 observations per cell in two-by-two table validating data recording method against gold standard; AUC criteria for high individual-level reporting accuracy: AUC $\geq 0.7$; IF criteria for low population-level bias: $0.75<\mathrm{IF}<1.25$. ${ }^{*}$ In the facility maternity register, essential newborn care is a composite indicator for (i) immediate initiation of breastfeeding and (ii) baby kept warm. 


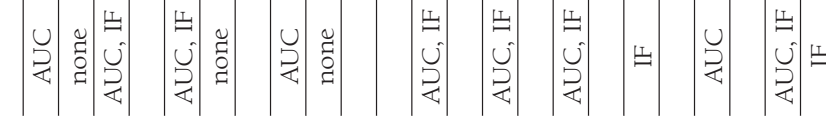

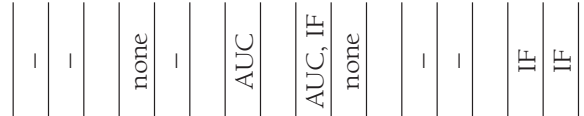

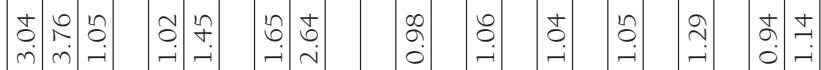

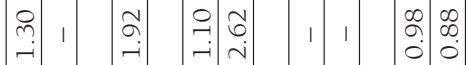

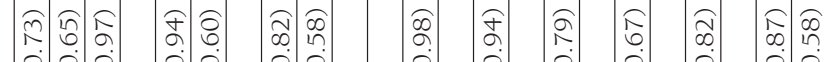

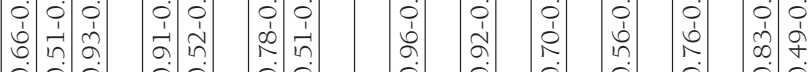
eे é

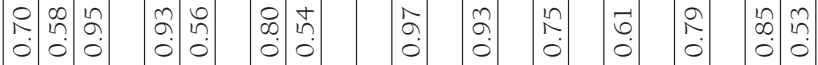

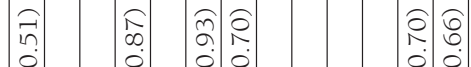
กิ่

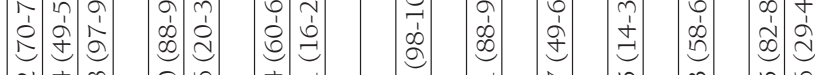

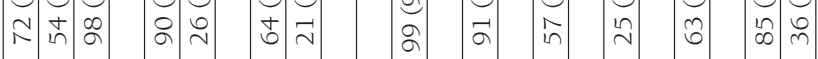

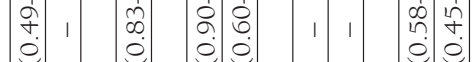
专约

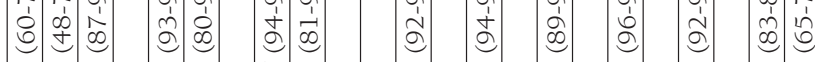

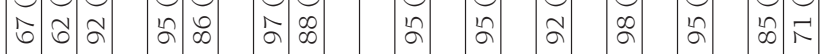

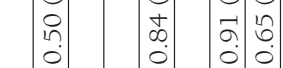
ț.

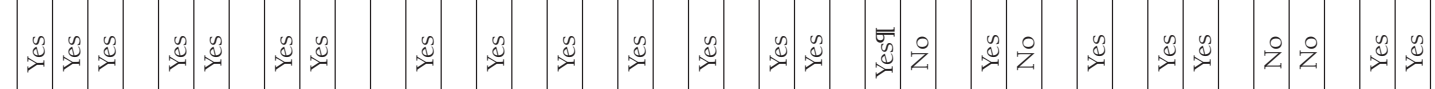
Nin

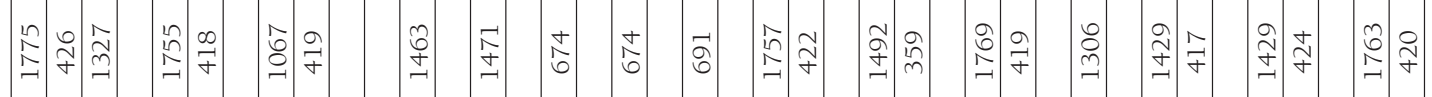
尹)

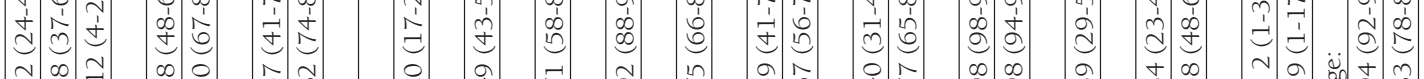

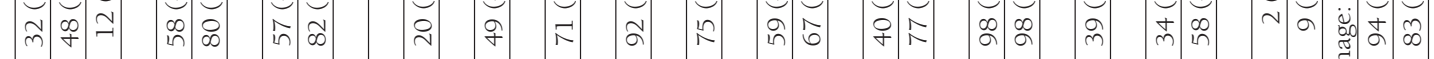

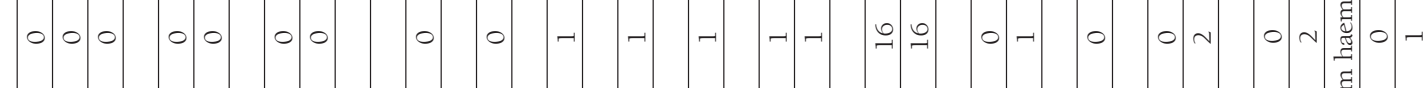

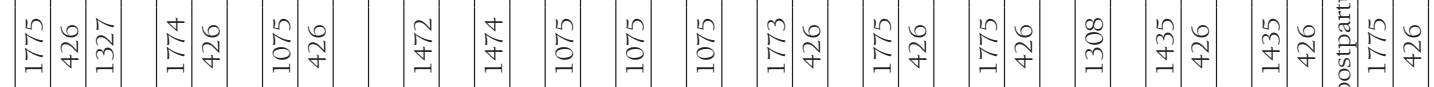

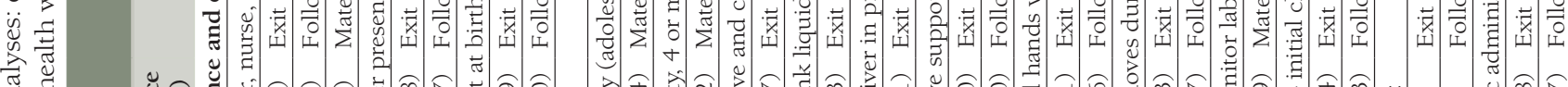

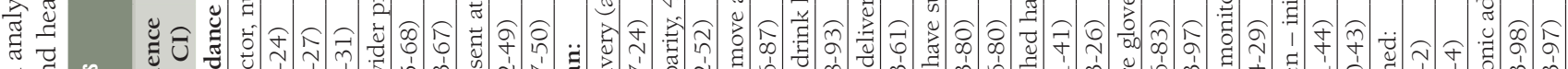

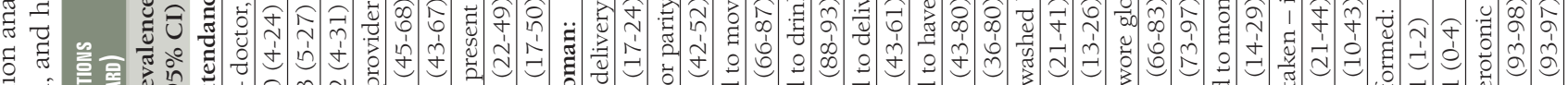

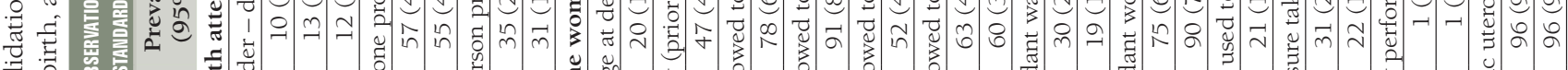

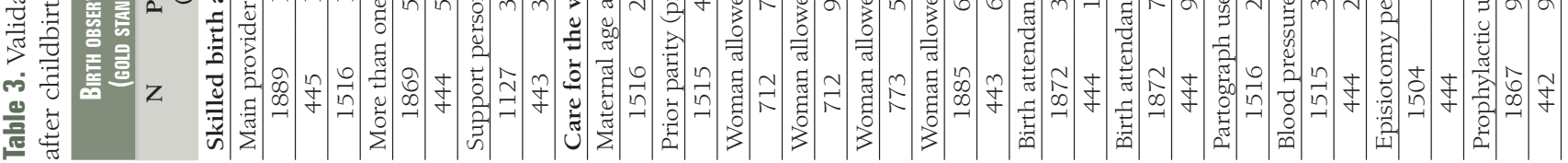




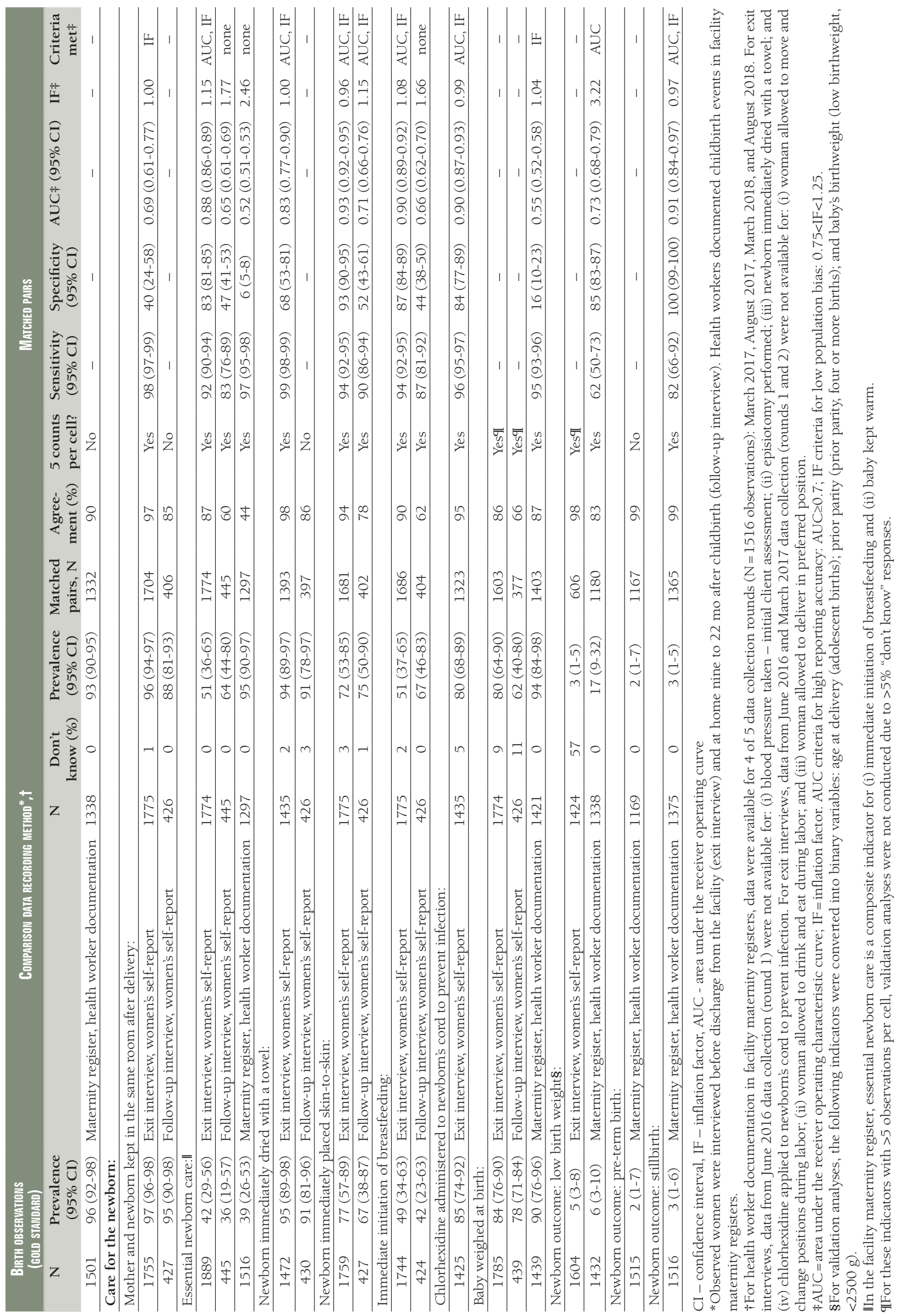


For additional immediate newborn care indicators assessed, women's recall during exit interviews had high overall validity for immediate drying of the newborn and the application of chlorhexidine on the newborn's cord. Women's recall of whether she and her newborn were kept in the same room after delivery nearly met the criteria for high overall validity, $A U C=0.69(95 \%$ confidence interval $(\mathrm{CI})=0.61-0.77$ ) and $\mathrm{IF}=1.00$. For whether the baby was weighed at birth, health worker documentation met criteria for low population-level bias.

For indicators related to low prevalence newborn outcomes, health worker documentation met high overall validity for whether a baby was stillborn and high individual-level accuracy for whether a newborn had low birthweight.

\section{DISCUSSION}

Providing high quality facility-based childbirth care with a skilled provider is essential for improving the health and survival of women and newborns. Accurate information on the care received is essential to monitoring progress. In Gombe state, where women predominantly seek childbirth care in rural primary health facilities, our study suggests that health worker documentation in facility registers, facility-level exit interviews, and household-level follow-up interviews can all contribute to accurate monitoring, but no individual method provided a broad understanding of the provision and experience of childbirth care.

Our validation of health worker documentation against a gold standard of birth observations differed from other accuracy studies of facility-based data. To date, studies assessed the extent to which data sources agreed when aggregated, reflecting the critical capacity to tally and report consistently between levels of the health system. Focusing on individual-level validity, health worker documentation had high validity (AUC $\geq 0.70$ and/or $0.75<\mathrm{IF}<1.25$ ) for select indicators about the main provider, maternal background characteristics, and newborn outcomes. Unsurprisingly, health workers were well-positioned to determine the provider's cadre and newborn outcomes such as stillbirths. Maternal background characteristics were also relatively stable data which could be verified during the antenatal period.

However, health worker documentation did not meet any validity criteria for essential newborn care, a composite indicator of immediate breastfeeding and keeping the baby warm. As noted earlier, the prevalence for essential newborn care within 30 minutes of birth documented by the health worker was $95 \%$ (95\% CI=90\%-97\%), whereas the observed prevalence for immediate breastfeeding and placing the newborn skin-to-skin within one hour of birth was only 39\% (95\% CI =26\%-53\%); health workers markedly overestimated the prevalence. Given the complexity of the essential newborn care definition, this may reflect the format of the documentation source which did not distinguish between care elements, as well as potential differences in interpretation between the observer and the health worker.

Our study adds new evidence to the validity of women's self-reports at different recall periods and focused on women who delivered in rural primary health facilities. We found that exit interviews had high validity for four immediate newborn care practices: drying the newborn with a towel; placing the newborn skin-to-skin; immediate breastfeeding; and applying chlorhexidine to a newborn's cord. In contrast to our study, two validation studies using hospital exit interviews in Mexico and Kenya did not report high validity for immediate drying of the newborn, placing the newborn skin-to-skin, and immediate breastfeeding $[18,19]$. Facility environment may explain part of the differences observed, which may in turn influence the frequency of "don't know" responses or the low specificity from a positive facility reporting bias $[18,19]$. For example, in our study, the practice of placing the newborn with the mother immediately after birth was 97\%, compared to 10\% in Mexico and 58\% in Kenya.

Similar to other validation studies, we found that women's self-reports during follow-up nine to 22 months after childbirth had low validity across indicators assessed. Placing a newborn skin-to-skin immediately after birth was the one exception, consistent with a follow-up study in Mozambique which included a nation-wide sample of rural and urban health facilities, but inconsistent with the Kenyan study [16,20]. One possible explanation for this being a memorable event for northeastern Nigerian women may be that the practice of immediate skin-to-skin contrasts with longstanding cultural beliefs on early bathing of newborns and the negative perceptions of vernix [43,44].

Indicators that met criteria for low population-level bias only, such as the administration of prophylactic uterotonic (exit, follow-up), permission to drink and eat during labor (exit), and baby weighed at birth (maternity register) had high prevalence, which masked a high false positive rate among the small number of clients that did not receive the service. Thus, we recommend caution when interpreting these indicators and triangulation with other data sources. 
Our findings highlight the importance of expanding the sources of data for monitoring the content of childbirth care. In addition to standard household surveys, monitoring of facility-based childbirth care should consider drawing from and linking multiple data sources including routine health facility data and exit interviews with recently delivered women. Facility-based routine data, such as registers, and exit interviews are useful sources for determining an accurate numerator when monitoring facility-based care; linkages to population-level data are still critical to determine the denominators for population in need and underserved subgroups [13]. At a global level, as greater emphasis is placed on respectful maternity care and the clients' experience of care, exit interviews are being included in the monitoring frameworks for assessing the quality of facility-based care [45]. Further, recent calls for greater investment in routine health information systems, if successful, would allow for monitoring beyond the global- and national-levels, as routine data are available at a greater level of disaggregation and frequency $[13,46,47]$.

The limitations of exit interviews and routine data still need careful consideration, however. Facility registers capture limited information about service delivery and, hence, provide a narrower but more frequent picture of quality of care. Health worker documentation and exit interviews are susceptible to reporting biases, whereby health workers record information only for the services they provide and women report receiving an intervention because of social desirability bias or a higher quality of care that might be assumed with a facility delivery $[17,18]$.

Among the strengths of this study was the use of birth observations as the gold standard which was compared to facility exit interviews, household follow-up interviews, and health worker documentation in maternity registers. The longitudinal study design allowed us to assess the validity of women's self-reports for different recall periods: before discharge from a facility and at nine to 22 months after childbirth, which more closely reflects the recall period and interviewing conditions of household surveys. Further, this study was novel as this setting was predominantly rural, based in the primary health care context, and included validation of health worker documentation in maternity registers. Among the limitations of the study, our findings primarily reflect the reporting accuracy of women who seek facility-based care. Further, women participating in household surveys are not usually interviewed twice; however, individual-level reporting accuracy decreased in our study which is different from what we would expect for repeated measurements. The gold standard could be susceptible to error from incorrect observer interpretation, errors in data recording, or changing behaviour because of the Hawthorne effect, even in the presence of quality control mechanisms [48]. Even with pre-testing, the questions in the exit and follow-up interviews may not have been interpreted as intended. Further, some observed indicators had such high or low coverage and were unsuitable for validation analyses. Finally, while not strictly a limitation, relatively stringent cut-off criteria were chosen for AUC and IF to align with other studies [23].

\section{CONCLUSION}

The childbirth process presents a time of great risk of death for women and newborns. Health worker documentation, facility-level exit interviews, and household-level follow-up interviews with women after childbirth each have a role to play in the accurate monitoring of facility-based childbirth care to improve the health and survival of women and their newborns.

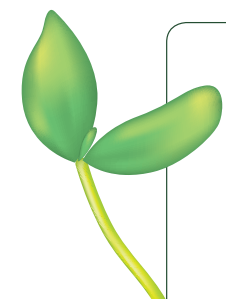

Acknowledgements: We are grateful to the women who participated in the study and shared their experiences. We appreciate the team who conducted the facility and household data collection. We also thank Katharine McCarthy for her comments on an earlier draft of the manuscript.

Funding: This work was supported by the Bill $\&$ Melinda Gates Foundation. The funder of this study had no role in the study's design or conduct, data collection, analysis or interpretation of results, writing of the paper, or decision to submit for publication.

Authorship contributions: AAB, TM, EA conceived, designed, and carried out the analyses. AAB and TM composed the initial draft. EA, JRM, NU, AU, HF, AA reviewed the early drafts. All authors approved the final draft.

Competing interests: Mr Felix Habila and Mr Ahmed Audu are members of the Gombe State Primary Health Care Development Agency. All authors have completed the Unified Competing Interest form at www.icmje.org/coi_disclosure.pdf (available on request from the corresponding author), and declare no further conflicts of interest.

\section{Additional material}

Online Supplementary Document 
1 World Health Organization. WHO recommendations: intrapartum care for a positive childbirth experience. Geneva: World Health Organization; 2018.

2 Lawn JE, Blencowe H, Oza S, You D, Lee ACC, Waiswa P, et al. Every Newborn: progress, priorities, and potential beyond survival. Lancet. 2014;384:189-205. Medline:24853593 doi:10.1016/S0140-6736(14)60496-7

3 Trends in maternal mortality: 1990 to 2015: estimates by WHO, UNICEF, UNFPA, World Bank Group and the United Nations Population Division. Geneva: World Health Organization; 2015.

4 United Nations Inter-agency Group for Child Mortality Estimation. Levels \& Trends in Child Mortality: Report 2018, Estimates developed by the United Nations Inter-agency Group for Child Mortality Estimation. New York: United Nations Children's Fund; 2018.

5 United Nations Population Fund. The State of World Population 2018. New York: United Nations Population Fund; 2018.

6 Bhutta ZA, Das JK, Bahl R, Lawn JE, Salam RA, Paul VK, et al. Can available interventions end preventable deaths in mothers, newborn babies, and stillbirths, and at what cost? Lancet. 2014;384:347-70. Medline:24853604 doi:10.1016/ S0140-6736(14)60792-3

7 World Health Organization. Standards for improving quality of maternal and newborn care in health facilities. Geneva: World Health Organization; 2016.

8 GBD 2015 Maternal Mortality Collaborators. Global, regional, and national levels of maternal mortality, 1990-2015: a systematic analysis for the Global Burden of Disease Study 2015. Lancet. 2016;388:1775-812. Medline:27733286 doi:10.1016/S0140-6736(16)31470-2

9 Kruk ME, Gage AD, Arsenault C, Jordan K, Leslie HH, Roder-DeWan S, et al. High-quality health systems in the Sustainable Development Goals era: time for a revolution. Lancet Glob Health. 2018;6:e1196-252. Medline:30196093 doi:10.1016/S2214-109X(18)30386-3

10 Tunçalp Ö, Were WM, MacLennan C, Oladapo OT, Gülmezoglu AM, Bahl R, et al. Quality of care for pregnant women and newborns-the WHO vision. BJOG. 2015;122:1045-9. Medline:25929823 doi:10.1111/1471-0528.13451

11 Hancioglu A, Arnold F. Measuring coverage in MNCH: Tracking progress in health for women and children Using DHS and MICS household surveys. PLoS Med. 2013;10:e1001391. Medline:23667333 doi:10.1371/journal.pmed.1001391

12 Short Fabic M, Choi Y, Bird S. A systematic review of Demographic and Health Surveys: data availability and utilization for research. Bull World Health Organ. 2012;90:604-12. Medline:22893744 doi:10.2471/BLT.11.095513

13 Countdown to 2030 Collaboration. Countdown to 2030: tracking progress towards universal coverage for reroductive, maternal, newborn, and child health. Lancet. 2018;391:1538-48. Medline:29395268 doi:10.1016/S0140-6736(18)30104-1

14 National Population Commission of Nigeria and ICF. Nigeria demographic and health survey 2013. Abuja, Nigeria, and Rockville, Maryland, USA: NPC/Nigeria and ICF International; 2014

15 National Bureau of Statistics and United Nations Children's Fund. Multiple Indicator Cluster Survey 2016-17, Survey Findings Report. Abuja, Nigeria: National Bureau of Statistics and United Nations Children's Fund; 2017.

16 Stanton CK, Rawlins B, Drake M, dos Anjos M, Cantor D, Chongo L, et al. Measuring coverage in MNCH: Testing the validity of women's self-report of key maternal and newborn health interventions during the peripartum period in Mozambique. PLoS One. 2013;8:e60694. Medline:23667427 doi:10.1371/journal.pone.0060694

17 Liu L, Li M, Yang L, Ju L, Tan B, Walker N, et al. Measuring coverage in MNCH: A validation study linking population survey derived coverage to maternal, newborn, and child health care records in rural China. PLoS One. 2013;8:e60762. Medline:23667429 doi:10.1371/journal.pone.0060762

18 Blanc AK, Diaz C, McCarthy KJ, Berdichevsky K. Measuring progress in maternal and newborn health care in Mexico: validating indicators of health system contact and quality of care. BMC Pregnancy Childbirth. 2016;16:255. Medline:27577266 doi:10.1186/s12884-016-1047-0

19 Blanc AK, Warren C, McCarthy KJ, Kimani J, Ndwiga C. RamaRao S. Assessing the validity of indicators of the quality of maternal and newborn health care in Kenya. J Glob Health. 2016;6:010405. Medline:27231541 doi:10.7189/ jogh.06.010405

20 McCarthy KJ, Blanc AK, Warren CE, Kimani J, Mdawida B, Ndwidga C. Can surveys of women accurately track indicators of maternal and newborn care? A validity and reliability study in Kenya. J Glob Health. 2016;6:020502. Medline:27606061 doi:10.7189/jogh.06.020502

21 Chang KT, Mullany LC, Khatry SK, LeClerq SC, Munos MK, Katz J. Validation of maternal reports for low birthweight and preterm birth indicators in rural Nepal. J Glob Health. 2018;8:010604. Medline:29899981 doi:10.7189/jogh.08.010604

22 Afulani PA, Phillips B, Aborigo RA, Moyer CA. Person-centred maternity care in low-income and middle-income countries: analysis of data from Kenya, Ghana, and India. Lancet Glob Health. 2019;7:e96-109. Medline:30554766 doi:10.1016/ S2214-109X(18)30403-0

23 Munos MK, Blanc AK, Carter ED, Eisele TP, Gesuale S, Katz J, et al. Validation studies for population-based intervention coverage indicators: design, analysis, and interpretation. J Glob Health. 2018;8:020804. Medline:30202519 doi:10.7189/ jogh.08.020804

24 Moller A-B, Newby H, Hanson C, Morgan A, El Arifeen S, Chou D, et al. Measures matter: A scoping review of maternal and newborn indicators. PLoS One. 2018;13:e0204763. Medline:30300361 doi:10.1371/journal.pone.0204763

25 Mavimbe JC, Braa J, Bjune G. Assessing immunization data quality from routine reports in Mozambique. BMC Public Health. 2005;5:108. Medline:16219104 doi:10.1186/1471-2458-5-108

26 Bosch-Capblanch X, Ronveaux O, Doyle V, Remedios V, Bchir A. Accuracy and quality of immunization information systems in forty-one low income countries. Trop Med Int Health. 2009;14:2-10. Medline:19152556 doi:10.1111/j.13653156.2008.02181.x 
27 Mphatswe W, Mate KS, Bennett B, Ngidi H, Reddy J, Barker PM, et al. Improving public health information: a data quality intervention in KwaZulu-Natal, South Africa. Bull World Health Organ. 2012;90:176-82. Medline:22461712 doi:10.2471/BLT.11.092759

28 Roomaney RA, Pillay-van Wyk V, Awotiwon OF, Nicol E, Joubert JD, Bradshaw D, et al. Availability and quality of routine morbidity data: review of studies in South Africa. J Am Med Inform Assoc. 2017;24:e194-206. Medline:27357829

29 Gimbel S, Mwanza M, Nisingizwe MP, Michel C, Hirschhorn L. Improving data quality across 3 sub-Saharan African countries using the Consolidated Framework for Implementation Research: results from the African Health Initiative. BMC Health Serv Res. 2017;17 Suppl 3:828. Medline:29297401 doi:10.1186/s12913-017-2660-y

30 Gimbel S, Micek M, Lambdin B, Lara J, Karagianis M, Cuembelo F, et al. An assessment of routine primary care health information system data quality in Sofala Province, Mozambique. Popul Health Metr. 2011;9:12. Medline:21569533 doi:10.1186/1478-7954-9-12

31 Day LT, Ruysen H, Gordeev VS, Gore-Langton GR, Boggs D, Cousens S, et al. "Every Newborn-BIRTH” protocol: observational study validating indicators for coverage and quality of maternal and newborn health care in Bangladesh, Nepal and Tanzania. J Glob Health. 2019;9:010902. Medline:30863542 doi:10.7189/jogh.09.010902

32 Nigeria Health Management Information System, version 2013: Gombe State facility deliveries, by facility type, January-December 2018 [Internet]. Nigeria District Health Information System, version 2. 2019.

33 World Health Organization. Strategies towards ending preventable maternal mortality (EPMM). Geneva: World Health Organization; 2015. Report No.: 9789241508483.

34 World Health Organization, United Nations Children's Fund. Every newborn: an action plan to end preventable newborn deaths. Geneva: World Health Organization; 2014.

35 Nigeria Federal Ministry of Health. Nigeria Health Management Information System, version 2013. Abuja, Nigeria; 2013.

36 London School of Hygiene \& Tropical Medicine. Informed Decisions for Action in Maternal and Newborn Health (IDEAS) Project 2018. Available: https://ideas.lshtm.ac.uk/. Accessed: 19 January 2019.

37 Exley JL, Umar N, Moxon S, Usman AU, Marchant T. Newborn resuscitation in Gombe State, northeastern Nigeria. J Glob Health. 2018;8:020420. Medline:30410739 doi:10.7189/jogh.08.020420

38 Nigeria DHIS2. Gombe State facility deliveries 2015-2018 [Internet]. Federal Ministry of Health. 2019 [cited May 29, 2019].

39 Gopstein D. clust.bin.pair: Statistical Methods for Analyzing Clustered Matched Pair Data 2018 Available: https:// cran.r-project.org/package=clust.bin.pair. Accessed: 14 January 2019.

40 Yang Z, Sun X, Hardin J. A note on the tests for clustered matched-pair binary data. Biom J. 2010;52:638-52. Medline:20976694 doi:10.1002/bimj.201000035

41 StataCorp. Stata, version 14.2. College Station, Texas, USA.

42 Vecchio TJ. Predictive value of a single diagnostic test in unselected populations. N Engl J Med. 1966;274:1171-3. Medline:5934954 doi:10.1056/NEJM196605262742104

43 George I, Ugboma H, Nyengidiki T. Newborn care in the delivering room: An observational study from Nigerian. Int J Trop Med. 2012;7:30-3. doi:10.3923/ijtmed.2012.30.33

44 Adejuyigbe EA, Bee MH, Amare Y, Omotara BA, Iganus RB, Manzi F, et al. "Why not bathe the baby today?": A qualitative study of thermal care beliefs and practices in four African sites. BMC Pediatr. 2015;15:156. Medline:26466994 doi:10.1186/s12887-015-0470-0

45 World Health Organization. Quality, Equity, Dignity. A Network for Improving Quality of Care for Maternal, Newborn and Child Health. Monitoring Framework: Working Document. Geneva: World Health Organization; 2017.

46 Bhattacharya AA, Umar N, Audu A, Felix H, Allen E, Schellenberg JRM, et al. Quality of routine facility data for monitoring priority maternal and newborn indicators in DHIS2: A case study from Gombe State, Nigeria. PLoS One. 2019;14:e0211265. Medline:30682130 doi:10.1371/journal.pone.0211265

47 Fryatt B, editor. Measurement and Accountability for Results in Health Summit, June 9-11, 2015; World Bank Headquarters, Washington, D.C.

48 McCambridge J, Witton J, Elbourne DR. Systematic review of the Hawthorne effect: New concepts are needed to study research participation effects. J Clin Epidemiol. 2014;67:267-77. Medline:24275499 doi:10.1016/j.jclinepi.2013.08.015 